\title{
Logical Coding \& Emotional Poetry: An exploration of poetic expression in the digital
}

\author{
Keywords \\ Digital Poetry, Ephemerality, Hidden Content, Non-linearity, Poetic Expression.
}

This practice-oriented design research investigates opportunities for meaningful poetic expression within a digital medium. It aims to create borndigital poems that tightly tie content to its form, prioritising the presentation and textual content of the poem. It hypothesises that the digital medium offers more opportunities for personal expression than in traditional print. Digital poems can be dynamic with animation, interactivity, and discovery. They can allow a poet to draw readers deeper into their world, where actions are reacted to and become part of the expression. However, these come with added complexities. This space can be intimidating for creatives as practitioners must learn coding languages to create interactive experiences. This project sits within digital poetry but borrows ideas from other realms of knowledge and practice, such as aesthetic expressivism and hypertext. The practice focuses on testing, reflecting, and articulating the poetic expression present in three distinct characteristics of the digital environment: ephemerality, hidden content, and non-linearity. These were initially derived from obsolescence of digital poetry, lack of orientation within hypertextual media, and definitions of hypertext. These characteristics are further investigated through prototypes, where the research tests techniques that reflect their qualities to create poetic experiences. Within this project, the researcher navigates differing worldviews: the poet and the technician. The former brings expressive and subjective lenses, while the latter introduces objectivity and attention to technical skills. Both are integral, and their relationship is at the heart of this research. At times, these voices were dissonant, yet there have been glimpses of symbiosis during the process. Arguably, finding ways to encourage the symbiosis between these two voices can ensure greater synergy and meaningful connections between the poem and its form. The research explores when poetic writing occurs in the process, and whether poetry or its form are prioritised. However, a design perspective can serve as a bridge between these two worlds. It is a role that already deals with both functionality and creativity. For this reason, prototypes are evaluated against a framework of paired communication design principles: movement and rhythm, emphasis and hierarchy, unity and contrast, and balance and space. This framework provides a basis to determine the suitability of techniques towards expressing a poetic idea or message. As a result, the research will map a range of workflows used for approaching digital poetry through the outlined characteristics of the medium. It is believed that without a clear understanding of what the digital space could offer, a poem could have no difference in its reading experience from its written print form, or practitioners could create a jarring and meaningless experience. The final design outcome of this research is an interactive digital poem themed around online relationships. It will provide an example of how these characteristics can be applied and used, highlighting possibilities for supporting expression through their relationships with the poem. 